\title{
Räume der Grenze - eine praxistheoretische Perspektive in den kulturwissenschaftlichen Border Studies
}

\section{Einleitung}

Die 2012 erschienene Einführung „Kultur - Von den Cultural Studies bis zu den Visual Studies“" stellt zentrale Forschungsfelder der Kulturwissenschaften vor. Diese verbindet das gemeinsame Interesse an Materialität, Medialität und Artefakten sowie eine sozial- und kulturkritische Sicht auf gesellschaftliche Prozesse. Als besonders kennzeichnend für die Studies hebt Stephan Moebius die „aus der Kultursoziologie herrührende praxistheoretische Perspektive“2 hervor, die das Soziale weder ausgehend von den Akteuren, noch von den Strukturen bestimmt. Den Ort des Sozialen bildet in praxistheoretischen Überlegungen das ,dazwischen' anzusiedelnde, strukturierende und beobachtbare Praktizieren von Kultur. Diese sich zunehmend durchsetzende Perspektivierung des Doing Culture kann auf „die Krise der Rational-Choice-Ansätze mit ihren empirisch unplausiblen handlungstheoretischen Modellannahmen“"3, auf die gesteigerte Aufmerksamkeit für das Materiale und auf die Krise der grand theories ${ }^{4}$ zurückgeführt werden. Denn während Letztere von einem nicht (mehr) tragfähigen Gesellschaftsbegriff ausgehen, privilegieren praxistheoretische Zugänge eine poststrukturalistische Lesart: Gesellschaft wird hier nicht als gegebene, stabile und geographisch verfasste Analyseeinheit angenommen, sondern als ein sich konstituierendes und zugleich dekonstituierendes Gebilde ${ }^{5}$. Das bedeutet, dass Gesellschaften in ihren Konstitutionsprozessen und damit über soziale Praktiken zu untersuchen sind. Diese sind dann zu thematisieren als „[...] fortlaufende Prozesse der Vergesellschaftung [...] - soziale Vollzüge in räumlich und zeitlich konkret bestimmbaren, materiell situierten und miteinander verknüpften Kontexten.“6

DaraufaufbauendwirdindiesemBeitrageineinderpraxistheoretischenPerspektive angelegte und mehrfach angesprochene ${ }^{7}$ - jedoch selten ausformulierte - Dimension des Sozialen beleuchtet. Gefragt wird, wie praxistheoretische mit raumtheoretischen

1 Moebius 2012a.

2 Ders. 2012b, 7.

3 Schmidt 2012, 11 .

4 Vgl. ebd.

5 Vgl. Bonacker 2008, 40.

6 Schmidt 2012, 12.

7 Vgl. ebd., 256; Schatzki 2010; ders. 2002; Reckwitz 2010, 186; ders. 2008, 91. 
Überlegungen verknüpft und in eine Heuristik der kulturwissenschaftlichen Raumanalyse überführt werden können. Die Fragestellung resultiert zum einen aus der mit Henri Lefebvre gewonnenen Einsicht „[...] (social) space is a (social) product“8, wonach Raum als ein emergenter Aspekt des Sozialen aufzufassen ist. Zum anderen interessiert diese Fragestellung, da der Raumbegriff trotz des spatial turn ${ }^{9}$ dort noch weitgehend unterbestimmt ist, wo er besonders herausgefordert ist: in den Border Studies, die sich als disziplinenübergreifendes Arbeitsfeld einer großen Bandbreite an Untersuchungsgegenständen zuwenden ${ }^{10}$. Dabei hat sich ein sozialkonstruktivistischer Forschungsstrang herauskristallisiert, der bordering-Prozesse als (machtvolle) Etablierungen, Verschiebungen oder Relativierungen von sozialen Demarkationen in den Blick nimmt ${ }^{\mathbf{1 1}}$. Der Raumbegriff spielt hier insofern eine Rolle, als dass Prozesse des de- und rebordering geographisch lokalisiert werden können und binär codierte Differenzierungen wie z. B. Wir/die Anderen oft als räumliche Metapher gefasst werden. Daneben ist ein pragmatischer Forschungsstrang zu unterscheiden, der sich zumeist an politischen Grenzen orientiert und die Dynamiken sowie Auswirkungen von geopolitischen Prozessen, supranationalen Organisationen und von grenzüberschreitenden Strömen (Waren, Dienstleistungen, Kapital, Menschen) untersucht. Die pragmatischen Border Studies privilegieren damit Untersuchungszusammenhänge, in denen politische Grenzen (als Barrieren oder Brücken) und national oder regional definierte Raumeinheiten eine zentrale Rolle spielen. Hier stellt sich das Problem, dass - entgegen der Einsicht Lefebvres - das Soziale zumeist vom Räumlichen her gedacht wird und dass die geopolitische Ordnung keinen theoretischen Platz für dauerhafte Grenzüberschreitungen zulässt. Angesprochen sind damit soziale Phänomene im Allgemeinen und in Grenzregionen im Besonderen, die transmigratorische Züge tragen. In anderen Worten: Phänomene, die sich im Grenzübertritt einrichten, die als zu nationalen Grenzen quer liegende Sozialzusammenhänge thematisiert werden können und die aus geopolitischer Sicht lediglich Formen des Zwischenkategorialen darstellen. Dazu zählen z. B. Ströme bzw. Personen, die in Grenzregionen regelmäßig und zirkulär eine Staatsgrenze überschreiten, um im Nachbarland einzukaufen, um dort Freizeitaktivitäten nachzugehen oder um dort zu arbeiten. Sie stellen das Ordnungsmodell der durch Staatsgrenzen unterscheidbaren geopolitischen Räume infrage.

Solche hier modellhaft als ,Grenzpendler` rubrizierten Phänomene werden im Folgenden mit Hilfe der praxistheoretischen Perspektive ihres zwischenkategorialen Status' enthoben und mögliche Ansatzpunkte ihrer empirischen Analyse unter räumlich-materialen Gesichtspunkten aufgezeigt. Dafür wird das Modell Räume der

8 Lefebvre 1991, 30.

9 Vgl. Günzel 2010; Döring u. Thielmann 2008; Bachmann-Medick 2007; Schroer 2006; Löw 2001.

10 z. B. Walter-Wastl 2011, 11-31; van Houtum u. van Naerssen 2002.

11 z. B. van Houtum, Kramsch, Zierhofer 2005. 
Grenze als eine mögliche Heuristik der kulturwissenschaftlichen Border Studies entwickelt, in dem raum- und praxistheoretische Perspektiven gewinnbringend konvergieren. Zunächst wird unter Rückgriff auf verschiedene Raumkonzepte und ihre Rezeption ein handlungstheoretisches Verständnis von Raum vorgestellt, das in seiner praxistheoretischen Reformulierung den Ausgangspunkt bildet für Räume der Grenze. $\mathrm{Zu}$ den Analysekategorien des Modells zählen soziale Praktiken, praktisches Wissen sowie physisch-materiale und sozial-strukturelle Aspekte, wie sie von ,Grenzpendlern' in grenzüberschreitenden Bezügen praxislogisch angeeignet und hervorgebracht werden.

\section{Raum: Substanz - Struktur - Bedeutung}

Den Ausgangspunkt der Überlegungen bildet der Raum. Auf die Frage, was Raum denn sei, antwortet der Sozialgeograph Peter Weichhart: „Das ist die ungelöste Grundfrage der Geographie. “12 Dieser Status des Begriffs ist ihm zufolge auf eine Vielzahl an Raumvorstellungen zurückzuführen, die innerhalb und außerhalb der Disziplin kursieren, unterschiedlich ausgelegt und zudem miteinander verschränkt werden. Zur Systematisierung des Feldes der Räume wird im Folgenden ein Überblick gegeben über drei zentrale Deutungen des Raumbegriffs, die in der weiteren Argumentation eine kritische Betrachtung des Handlungsbegriffs erforderlich machen.

Das absolut-substantialistische Raumkonzept als erste Deutung entwirft Raum als ein real existierendes Element der physisch-materialen Welt. Es schließt sowohl geographisch lokalisierbare Erdraumausschnitte als auch den von physisch-materialen Elementen abstrahierten Raum ein. Raum im Sinne der Erdoberfläche bezeichnet einen durch dominante Gegebenheiten spezifizierten und sichtbaren Ausschnitt der physischen Welt, wie z. B. den Mittelmeerraum oder einen Ballungsraum. Die Raumgrenzen werden hier in Orientierung an Merkmalen des zu bezeichnenden Erdausschnitts definiert und sind zumeist unscharf gezogen. Daneben ist Raum als dreidimensionale Ausdehnung im Sinne eines Behälters zu unterscheiden, in dem Objekte, Personen oder Ereignisse vorkommen. Dieses Verständnis formulierte Isaac Newton unter dem Eindruck der klassischen Mechanik im 18. Jh. folgendermaßen: „Der absolute Raum, der aufgrund seiner Natur ohne Beziehungen zu irgendetwas außer ihm existiert, bleibt sich immer gleich und unbeweglich." ${ }^{13}$ Raum wird hier eine eigene und statische Wesenhaftigkeit zugeschrieben, die unabhängig von anderen Objekten existiert. Es geht somit um „[...] jenes ,Ding‘, das übrig bleibt, wenn man gleichsam

12 Weichhart 2008, 75.

13 Newton 1988, zitiert in: Löw 2001, 25. 
aus einem Gebirgsraum das Gebirge herausnimmt. “14 Diese absolutistische Raumvorstellung hat sich in der Vergangenheit in vielen Wissenschaftsdisziplinen etabliert. So z. B. in der Geographie, in der Friedrich Ratzel im 19. Jh. das Lebensraumkonzept als Behältnis für Lebens-, Kultur-, Gesellschafts- und Wirtschaftsformen stark machte ${ }^{15}$. Die darin angelegte Naturdeterminiertheit des Sozialen prägte das Fach zum Teil noch bis in die zweite Hälfte des 20. Jh. und verweist auf die Idee, dass Raum auf die in ihm befindlichen Objekte und Menschen einwirke. In die Sozialwissenschaften hat das absolut-substantialistische Raumkonzept unter anderem mit der Annahme Eingang gefunden, dass in Nationalstaaten das Territorium, die Nation, der Staat und die Kultur zu einer - nach innen homogenen und inkludierend wirkenden und nach außen abgeschlossenen und exkludierend wirkenden - Einheit zusammenfielen. Dass die Ränder solcher „Raumgestalten“16 durchlässig sind und der Homogenitätsbzw. Schließungscharakter von Gesellschaften nicht haltbar ist, haben ,Grenzpendler ${ }^{6}$ schon lange vor der Internationalisierung der 1990er Jahre gezeigt. ${ }^{17}$ Dennoch wird das Containermodell erst unter dem Eindruck von Globalisierungsdynamiken verstärkt problematisiert. So wurde hinterfragt, ob die ,Insassen“ von Nationalcontainern tatsächlich als Agenten makrostruktureller Logiken angesehen werden können und welche Erklärung die Kongruenz von Territorium, Nation, Staat und Kultur tatsächlich (noch) liefern kann. Mit Konzepten wie „Denationalisierung“, „Deterritorialisierung “ oder „Weltgesellschaft“18 und der aufkommenden Enträumlichungsthese wandelte sich der Status des absolut-substantialistischen Raumbegriffs: Da sich das Soziale mittels moderner Technologien und Medien vom Raum emanzipiere, sei von einem wachsenden Bedeutungsverlust des Raums auszugehen. Die Enträumlichungsthese - als ein immer wiederkehrender Topos von technischen Neuerungen ${ }^{19}$ - versucht zwar die Containerraumvorstellung zu überwinden, gleichwohl bleibt sie konstitutiv in der Argumentation für das ,Verschwinden von Raum‘. Denn das geopolitische Ordnungsmodell der Nationalstaaten bleibt hier der Bezugspunkt von raumbezogenen Betrachtungen, wenngleich diese Ordnung unterlaufen wird aufgrund der mühelosen Überwindung naturräumlicher Distanzen und der „umspülenden und unterhöhlenden Ströme von Waren, Daten und Menschen“20. Die sozial- und kulturwissenschaftliche Analyse solcher ,enträumlichenden` Phänomene hat schließlich dafür sensibilisiert, dass der Raum keineswegs an seinem Ende angekommen ist. Vielmehr zeichnet sich durch Mobilität und Vernetzung ein neues Raumgreifen ab. Die damit aufgerufene Verräumlichungsthese hebt auf die vielfältigen, räumlichen

14 Weichhart 2008, 77.

15 Vgl. Ratzel 1966; Werlen 2009, 149.

16 Werlen 1997, 44.

17 Vgl. z. B. Wille 2012.

18 z. B. Mau 2007, 35f.

19 Vgl. Schroer 2008, 128.

20 Ebd., 135. 
Bezüge des Sozialen ab, die oftmals keinen nationalen Ordnungslogiken folgen, sondern quer zu nationalen Grenzen liegen und als soziale, virtuelle oder transnationale Räume beschreibbar sind. Ihnen gemeinsam ist eine sozialkonstruktivistische und relationale Perspektive, die das (wieder-)entdeckte Interesse am Raum - etwa als Space Studies ${ }^{21}$ - wesentlich befördert hat.

Das relational-konstruktivistische Raumkonzept bezieht sich ebenso wie der absolut-substantialistische Raumbegriff auf die physisch-materiale Welt, jedoch stehen hier die Eigenschaften derselben im Vordergrund. Raum als Relation rückt dann in den Blick, wenn die Anordnungen bzw. das Nebeneinander von physisch-materialen Elementen thematisiert wird, die in einem Erdraumausschnitt lokalisiert werden können. Das relationale Verständnis ist unter anderem auf Albert Einstein zurückzuführen, der mit der Relativitätstheorie die Vorstellung von Raum als einer übergeordneten Realität widerlegte. Er ging von der Lagerungsqualität der Körperwelt aus, womit sich Raum als eine Beziehungsstruktur zwischen Körpern und Artefakten darstellt. ${ }^{22}$ Raum wird also nicht länger unabhängig von einem Inhalt gedacht, sondern physisch-materiale Elemente werden hier konstitutiv. Damit bildet die Lagerungsqualität von Körpern und Artefakten einen Beschreibungsmodus für räumliche Verhältnisse, die in ihrer Relationalität veränderbar sind. Diese Auffassung wird zumeist dort (implizit) zu Grunde gelegt, wo Transaktionen, Beziehungen, Ströme (flows) oder Netzwerke betrachtet werden. So z. B. in der relationalen Wirtschaftsgeographie, die sich vom raumwirtschaftlichen Ansatz abwendet und das Räumliche über ein lokalisierbares Geflecht von sozio-ökonomischen Beziehungen erschließt. ${ }^{23}$ In den Politikwissenschaften ist die relational-konstruktivistische Perspektive in Integrationstheorien vorzufinden, wie z. B. im transnationalen Regionalismus. Er zielt auf einen europäischen Integrationsprozess ,von unten` ab durch grenzüberschreitende und interregionale Zusammenarbeit sowie durch transnationale Netzwerkbildung zwischen sogenannten subnationalen Einheiten. ${ }^{24}$ Auch in der Migrationssoziologie wird das Räumliche relational-konstruktivistisch gefasst, wenn durch (Trans-)Migrationsströme ein transnationaler sozialer Raum entsteht. Dieser wird vom Soziologen Ludger Pries definiert als

[...] relativ dauerhafte, auf mehrere Orte verteilte bzw. zwischen mehreren Flächenräumen sich aufspannende verdichtete Konfigurationen von sozialen Alltagspraktiken, Symbolsystemen und Artefakten. Sie sind [...] in verschiedenen Territorien bzw. locales verankert, die wiederum in andere sozialräumliche Einheiten - z. B. von nationalen Container-Gesellschaften - eingewoben sind. ${ }^{25}$

21 Vgl. Kajetzke u. Schroer 2012.

22 Vgl. Einstein 1960, zitiert in: Löw 2001, 34.

23 Vgl. Bathelt u. Glückler 2003.

24 Vgl. Schmitt-Egner 2005, 148.

25 Pries 2008, 195. 
Die exemplarisch genannten Ansätze nehmen translokale Beziehungen in den Blick und leiten daraus räumliche Strukturen ab. Physisch-materiale Elemente und ihre Lagebeziehungen sind somit nicht mehr in einen umgebenden Behälter verbannt, sondern sie machen den Raum aus. Die relational-konstruktivistische Perspektive eröffnet so eine erste Möglichkeit ,Grenzpendler' auch in den pragmatischen Border Studies raumtheoretisch zu denken, können ihre Raumkonstruktionen doch anhand der in grenzüberschreitenden Aktivitäten in Anordnung gebrachten Körper und Artefakte rekonstruiert werden. Bei der Erfassung des grenzüberschreitenden Spacing ${ }^{26}$ besteht jedoch die Gefahr, dass die Autonomie von ,Grenzpendlern“ überhöht wird und der Einfluss von (natur-)räumlichen Bedingungen, von nationalen Grenzziehungen und der damit verbundenen Implikationen auf das Hervorbringen von räumlichen Strukturen aus dem Blick geraten. Kajetzke und Schroer plädieren daher für die Berücksichtigung beider Aspekte: „die Wirkmacht räumlicher Strukturen“ und „die schöpferische Kraft der Individuen“27. Ferner ist mit der relational-konstruktivistischen Perspektive die Gefahr verbunden, dass Raum lediglich anhand von Transaktionsströmen, Beziehungsverflechtungen oder Netzwerkkonfigurationen beschreibend nachgezeichnet wird und die Qualität dieser Strukturen - als eine sinnhaft konstituierte Räumlichkeit - vernachlässigt bleibt. Die deskriptive Dimension von Raum kann zwar von der qualitativen Dimension unterschieden werden, sie bilden jedoch zwei miteinander verschränkte Aspekte der Raumproduktion und ihrer Analyse. Dies wird mit dem sozial-konstitutiven Raumkonzept deutlich, das den Schwerpunkt weniger auf die räumliche Substanz oder auf die räumlichen Strukturen legt, sondern auf die Bedeutungsebene von Raum.

Im sozial-konstitutiven Raumverständnis wird die entwickelte Position, dass das Räumliche keine eigene Wesenhaftigkeit besitzt und vom Sozialen her zu denken ist, um die Sinnebene ergänzt. Zunächst ist auf eine erlebnisräumliche Bedeutung einzugehen, die sich auf die subjektive Wahrnehmung von Lagestrukturen bezieht. Es geht dabei um einen erlebten Raum, wie z. B. ,das Studentenviertel', in dessen Repräsentation bestimmte Deutungen, Bewertungen und Erinnerungen einfließen. Einen empirischen Zugriff auf solche Repräsentationen erlaubt die Mental-Map-Forschung, die versucht „[...] festzustellen, wie Individuen ihre räumliche Umwelt subjektiv in ihrem Bewusstsein abbilden.“28 Über solche Abbilder, die Löw als Syntheseleistung - im Sinne einer mentalen Zusammenfassung von Subjekten und Artefakten zu Räumen thematisiert ${ }^{29}$, wäre ein erster an Sinn orientierter Zugang zu Räumen möglich, wie sie ,Grenzpendler‘ in grenzüberschreitenden Bezügen hervorbringen.

26 Martina Löw führt die Kategorie des Spacing ein und bezeichnet damit den Prozess der Raumkonstruktion durch das Platzieren von sozialen Gütern und Menschen (vgl. Löw 2001, 158).

27 Kajetzke u. Schroer 2010, 203.

28 Werlen 2004, 286.

29 Vgl. Löw 2001, 159. 
Eine weitere Deutung des sozial-konstitutiven Raumbegriffs fokussiert auf die tätige und sinnhafte Auseinandersetzung des Subjekts mit seiner sozialen und materialen Umwelt. Ausgegangen wird davon, dass Körpern und Artefakten keine Bedeutungen eingeschrieben sind, sondern sie erst im Umgang bedeutsam und damit räumlich voraussetzungsvoll werden: „Sie [Raum oder Materialitäten] werden erst in Handlungsvollzügen unter bestimmten sozialen Bedingungen bedeutsam. “30 Das Erkenntnisinteresse liegt hier auf der Frage, wie Raum in seiner materialen und sinnhaften Dimension durch Subjekthandeln konstituiert wird. Damit rücken Prozesse der Raumproduktion in den Blick, die einerseits die Rekonstruktion von Lagestrukturen erfordern und andererseits über die Rekonstruktion von Sinnstrukturen zugänglich werden, die im ,anordnenden“ Handeln wirksam und hervorgebracht werden.

Diese Sicht auf Raum wurde im deutschsprachigen Raum von dem Geographen Benno Werlen in den 1980er Jahren stark gemacht. In der „Sozialgeographie alltäglicher Regionalisierungen“31 sollte das Soziale nicht länger in räumliche Kategorien ,zergliedert‘, sondern der Konstitutionsprozess von räumlichen Verhältnissen thematisiert werden. Der Enträumlichungsthese folgend argumentiert Werlen, dass Raum aufgrund der Entankerungsmechanismen (disembedding) der Spätmoderne keinen bestimmenden Faktor mehr bilde, weshalb die Erklärung von Phänomenen in physisch-materialen Kategorien zu kurz greife:

Mit der Durchdringung der lokalen Kontexte durch globalisierte Wissensbestände wird es immer schwieriger zu behaupten, jemand würde auf diese oder jene Art handeln, weil er einen bestimmten Herkunftsort aufweist. Die räumliche Konnotation der Herkunft oder aktuellen Lebensumstände erklärt in sozial-kultureller Hinsicht nichts mehr oder zumindest immer weniger. ${ }^{32}$

Raumanalysen sollten sich im Sinne der Verräumlichungsthese vielmehr auf das „Geographie-Machen“"33 der Subjekte bzw. auf die von ihnen „praktisch inszenierten Geographien““34 konzentrieren. Als analytischen Ausgangspunkt für dieses Raumgreifen führt Werlen das Alltagshandeln der Subjekte an, um den darin hervorgebrachten räumlichen Verhältnissen nachzuspüren. ${ }^{35}$ Mit Raum werden dabei in deskriptiver Hinsicht „[...] die unterschiedlichen Relationierungen der körperlichen Subjekte mit anderen physisch-materiellen Gegebenheiten [...] zum Ausdruck“"36 gebracht; in qualitativer Hinsicht bezeichnet Raum die im Rahmen von Relationierungen hervorgebrachten Sinnzuschreibungen und Sinndeutungen der Subjekte. Damit sind die

30 Werlen 1999, 223.

31 Ders. 1997; ders. 2010.

32 Ders. 2008a, 379.

33 Ders. 2007a, 28.

34 Lippuner 2005, 31.

35 Vgl. Werlen 2007a, 16 u. 231.

36 Ders. 2007b, 10. 
- lediglich analytisch zu trennenden - Aspekte des sozial-konstitutiven Raumbegriffs genannt: Zum einen die relationalen Lagestrukturen von Artefakten und Körpern, die von Subjekten im Alltagshandeln hervorgebracht werden; zum anderen die Sinndeutungen und Sinnzuschreibungen gegenüber der materialen und sozialen Welt, die in Alltagshandeln einfließen und sozial wirksam werden.

Das sozial-konstitutive Raumkonzept mit seinen Bezugnahmen auf den relational-konstruktivistischen Raumbegriff eröffnet für die Border Studies einen um die Sinnebene erweiterten und handlungstheoretischen Zugang zu ,Grenzpendlern'. Die vorausgesetzte Gemachtheit von Raum, der sich auch quer zu nationalen Grenzen aufspannen kann, umgeht den theoretischen Konflikt mit nationalstaatlichen Ordnungen und vermag dem bislang unsichtbaren Zwischenkategorialen eine empirische Gestalt zu geben. Es ist das grenzüberschreitende Geographie-Machen der Subjekte, das nun den Ausgangspunkt der Raumanalyse bildet und eine weiterführende Beschäftigung mit dem Handlungsbegriff notwendig macht.

\section{Handeln: Zwecke - Normen - Wissen - Körper}

Anknüpfend an das entwickelte handlungstheoretische Raumverständnis wird im Folgenden gefragt, wie ein Handlungsbegriff beschaffen sein muss für die Beschreibung und Analyse von subjektzentrierten Raumkonstitutionen in grenzüberschreitenden Bezügen. Ausgangspunkt der Überlegungen ist weiter Werlen, der das Geographie-Machen als eine „Tätigkeit im Sinne eines intentionalen Aktes“ definiert, „bei dessen Konstitution sowohl sozial-kulturelle, subjektive wie auch physisch-materielle Komponenten bedeutsam sind. " ${ }^{37}$ Werlen führt damit Intentionen und Zwecke in den Blick, auf welche die Subjekte ihre Tätigkeiten ausrichten, im Rahmen derer wiederum physisch-materiale Elemente bestimmte Bedeutungen erlangen. Dieser Prozess orientiert sich „mehr oder weniger bewusst an einem intersubjektiven [...] Bedeutungszusammenhang“ im Sinne eines ,gesellschaftlich und kulturell vorbereitete[n] Orientierungsraster[s]“, das „unabhängig vom einzelnen Handelnden besteht“38. Das hier vorgelegte Handlungsverständnis knüpft in seiner Zweck- und Regelorientierung an klassische Ansätze der Handlungserklärung an, die mit Blick auf Handeln in grenzüberschreitenden Bezügen zu diskutieren sind.

Die zweckorientierten Handlungsansätze (z. B. Max Weber, Vilfredo Pareto) als erster Diskussionsgegenstand sind besonders im Feld des Ökonomischen vorzufinden und umfassen solche Theorien, die individuelles Handeln aus Interessen und Kosten-Nutzen-Erwägungen heraus erklären. Für den homo oeconomicus wird somit

37 Werlen 2008b, 282.

38 Ebd., 287. 
eine rationale Handlungsorientierung vorausgesetzt, nach der ein Individuum sein Verhalten - auf Grundlage von Informationen und Fähigkeiten zur Zielerreichung bewusst auf bestimmte Zwecke ausrichtet. Das Soziale bildet dann die Summe der aufeinander abgestimmten Einzelhandlungen, die sich in Interaktionssituationen herausbildet. ${ }^{39}$,Grenzpendlern' sind durchaus gewisse Interessen und Kosten-Nutzen-Kalkulationen zu unterstellen, ist grenzüberschreitendes Geographie-Machen doch oftmals auf persönliche Vorteilsmaximierungen zurückzuführen aufgrund von Preisunterschieden, (Netto-)Einkommensunterschieden oder unterschiedlichen Angeboten im Freizeitbereich dies- und jenseits einer nationalen Grenze. ${ }^{40}$ Dennoch greift ein lediglich an Zwecken und Interessen orientierter Handlungsbegriff zu kurz, ist doch insbesondere für grenzüberschreitendes Alltagshandeln von keiner vollständigen Information zur rationalen Abwägung und Erwartbarkeit der Zielerreichung auszugehen.

Die normorientierten Ansätze (z. B. Talcott Parsons, Robert Merton, Émile Durkheim), die mit der Modellfigur des homo sociologicus wiedergegeben werden, erklären die Geordnetheit von Handeln mit Erwartungen, Werten und Rollen. Damit treten an die Stelle der Handlungsziele kollektiv geteilte Handlungsnormen sowie die Fähigkeiten der Individuen zur Normberücksichtigung. Das Soziale wird so nicht mehr als Summe aggregierter Einzelhandlungen thematisiert, sondern als ein stabiler normativer Konsens, der eine intersubjektive Koordination von potentiell sich widersprechenden Handlungen ermöglicht und reguliert. ${ }^{41}$ Wird dieses Regulativ auf grenzüberschreitendes Handeln übertragen, so sind zunächst die vorausgesetzten normativ-integrierten Kollektive zu problematisieren. Denn grenzüberschreitendes Geographie-Machen erstreckt sich dann mindestens über zwei solche Kollektive dies- und jenseits einer nationalen Grenze, für die in der Regel jeweils ein anderer normativer Konsens über legitimes und illegitimes Handeln angenommen wird. Für ,Grenzpendler' würde sich dann die Frage nach der Normberücksichtigung, welche die Kenntnis der sozialen Regeln beiderseits der Grenze voraussetzt, in besonderem Maße stellen. Diese Frageperspektive impliziert jedoch, dass das Soziale vom Raum her - und nicht umgekehrt - gedacht wird und dass das kreativ-produktive Moment einer möglichen Verschiebung von Handlungsroutinen unberücksichtigt bleibt. Damit erweisen sich normorientierte Ansätze bereits an dieser Stelle als wenig tragfähig, um Alltagshandeln zu erklären, das besonders in grenzüberschreitenden Bezügen von Diskontinuitäten, Mehrdeutigkeiten sowie kulturellem Wandel gekennzeichnet ist. ${ }^{42}$

39 Vgl. Reckwitz 2004, 307f., und ders. 2003, 287.

40 Vgl. Wille 2012, 219ff.

41 Vgl. Reckwitz 2003, 287.

42 Vgl. Boeckler 2012, 48. 
Kulturtheoretisch-wissensorientierte Ansätze (z. B. Alfred Schütz, Claude LéviStrauss, Roland Barthes) erklären Handeln nicht über individuelle Zwecke oder kollektive Normen, sondern über Wissensordnungen. Diese bilden ein Kriterium für die symbolische Organisation der Wirklichkeit und für Bedeutungszuweisungen, an denen Subjekte ihr Handeln orientieren. Dementsprechend wird auch für den homo significans eine Geordnetheit des Handelns vorausgesetzt, wobei die Regeln nicht normativ, sondern kognitiv gefasst und in Prozessen der symbolischen Repräsentation sowie der Sinnzuweisungen regulativ wirksam werden. Handeln wird somit an kulturellen Codes, Symbolsystemen und Bedeutungen festgemacht, nach denen die Subjekte die Wirklichkeit - in aufeinander abgestimmter Weise - interpretieren und reproduzieren. Dabei wird die Handlungswirksamkeit von Zwecken und sozialen Normen nicht ausgeblendet, sondern es wird versucht zu rekonstruieren, weshalb Subjekte gewisse Zwecke für wünschenswert und bestimmte Normen für situationsadäquat halten. ${ }^{43}$ Werden kognitive Wissensordnungen als Regulativ des grenzüberschreitenden Geographie-Machens herangezogen, so ergeben sich aufgrund ihres intersubjektiv und stabil gedachten Charakters erneut Probleme. Dies erläutert Alfred Schütz (1972) anschaulich mit dem Fremden: Dieser gibt sich als solcher zu erkennen durch die von ihm angelegten ,originären' Normalitätserwartungen bzw. Wissensordnungen. Der Status des Fremden ist erst dann überwunden, wenn er die entsprechenden Hintergrundannahmen und Relevanzsysteme der aufnehmenden Kultur ,erlernt ${ }^{\star}$ hat. Schütz optiert demnach bei der Begegnung unterschiedlicher Wissensordnungen für das Modell der Assimilation, das auf ein völliges Aufgehen in den normalitätsstiftenden kulturellen Codes der aufnehmenden Kultur abstellt und weder Diskontinuitäten von Handlungsroutinen noch eine Pluralität von (grenzüberschreitend zirkulierenden) Sinnangeboten einräumt. Mit Blick auf ,Grenzpendler` kann zwar von keiner aufnehmenden Kultur gesprochen werden, gleichwohl ist im grenzüberschreitenden Geographie-Machen von interpretativen Unbestimmtheiten auszugehen, die in interkulturalistischer Manier - auf unterschiedliche Symbolsysteme und Wissensstrukturen zurückgeführt werden könnten und in denen das hier unberücksichtigte kreativ-produktive Moment von Handeln dann besonders herausgefordert wäre.

Neben den genannten Problemen der vorgestellten Ansätze der Handlungserklärung sind weitere Aspekte zu nennen, die für die raumsensible Analyse von Alltagshandeln in grenzüberschreitenden Bezügen problematisch sind. Dazu zählen die Regelsysteme und Bedeutungszusammenhänge, die außerhalb des Handelns angenommen und als normative bzw. kognitive Muster im Innern der Subjekte wirksam werden. Diese Sichtweise führt einerseits zu der - besonders in grenzüberschreitenden Bezügen - problematischen Frage nach der ,ordentlichen' Ausführung ,gültiger Regel- und Symbolsysteme und marginalisiert Subjektleistungen. Andererseits gerät damit lediglich die mentale Dimension von Handeln in den Blick; das beobacht-

43 Vgl. Reckwitz 2004, 314-331, und ders. 2003, $288 f$. 
bare Handeln und seine Materialisierungen bleiben hier vernachlässigt. Praxistheoretische Ansätze hingegen berücksichtigen nicht nur den körperlichen Vollzug von Handeln, sondern operieren mit dem Praktikenbegriff und führen damit eine Reihe von theoretischen und für die Fragestellung des Beitrags vielversprechenden Implikationen ein.

Praxistheoretische Ansätze: Die praxistheoretischen Ansätze (z. B. Pierre Bourdieu, Anthony Giddens, Theodore Schatzki, Bruno Latour) als sozialkonstruktivistische Variante der Kulturtheorien entwickeln - mit ihren jeweiligen Schwerpunktsetzungen - eine Perspektive auf menschliche Aktivität, die kulturelle Kontingenz und die körperliche Auseinandersetzung des Subjekts mit seiner sozialen und materialen Umwelt einschließt. Handeln wird dabei nicht als punktuelle Einzelaktivität mit ,dahinterliegenden' Zwecken und Normen verstanden, sondern als eine Verkettung von sich reproduzierenden und aktualisierenden Praktiken, die material verankert und sozial verstehbar sind. Unter soziale Praktiken werden dann „,...] körperliche Darstellungen ,praktischer Vernunft“ (Mauss) und ,sinnhafter Verstehensleistungen““ gefasst, die durch „spezifische Formen des impliziten Wissens“ zusammengehalten werden und im Zusammenspiel stehen mit gegenständlichen Artefakten und natürlichen Dingen. ${ }^{44}$ Das hier angesprochene Wissen besitzt dabei weder eine übersubjektive Existenz, noch ist es im Bewusstsein des homo in praxi ,eingelagert‘. Vielmehr wird es dem praktischen Vollzugsgeschehen zugeordnet, wo es unmittelbar wirksam wird und den Rahmen dafür bildet, „[...] wie konkrete Dinge in einer Praktik zu interpretieren und wie sie praktisch zu handhaben sind, welcher ,praktische Sinn` entwickelbar ist. “ ${ }^{45}$ Demzufolge ist das Soziale hier weder in der normativen Abgestimmtheit der Handlungen, noch in der Intersubjektivität kultureller Codes zu suchen, sondern in den körperlichen Vollzügen der Praktiken, in denen soziale Ordnungen praxislogisch hervorgebracht, verändert und reproduziert werden. Soziale Praktiken bilden somit ein kontingentes und strukturierendes Moment der sozialen Wirklichkeit, in denen sowohl Brüche möglich sind als auch typische Aktivitäten sich immer wieder aufs Neue herausbilden. ${ }^{46}$

Für die handlungstheoretische Raumanalyse in grenzüberschreitenden Bezügen scheinen praxistheoretische Ansätze gewinnbringend zu sein. Sie bieten Anknüpfungspunkte für die theoretische und empirische Berücksichtigung von Körpern und Artefakten, die für raumtheoretische Anschlüsse nützlich sind. Des Weiteren wird mit der Betonung der Vollzugsdimension von Handeln die vermeintliche übersubjektive Existenz von Regelsystemen und Bedeutungszusammenhängen überwunden, denn praktisches Wissen - das nicht auf die Frage der Normberücksichtigung, der räumlichen Gültigkeitsbereiche oder auf die Idee der sozial integrierten Kollektive

44 Moebius 2008, 59 u. 61.

45 Reckwitz 2010, 193.

46 Vgl. Schmidt 2012, 10. 
abstellt - wird in den körperlichen Praktiken performativ hervorgebracht. Somit steht hier nicht Wissen als eine Eigenschaft von ,Grenzpendlern' oder ein räumlicher Gültigkeitsbereich für bestimmte Wissensstrukturen dies- und jenseits einer nationalen Grenze im Zentrum, sondern die Frage, welches Wissen in bestimmten sozialen Praktiken wirksam, aktualisiert und hervorgebracht wird bzw. rekonstruiert werden kann. ${ }^{47}$ Demzufolge sind Wissen und Handeln nicht voneinander getrennt, sondern miteinander verschränkt zu denken, um Erklärungen für Subjekthandeln und soziale Ordnungen zu ermitteln. Diese Sichtweise relativiert handlungsanleitende Regelsysteme und symbolische Ordnungen, wird praktisches Wissen - als körperliche Artikulation - doch als kontingent und wandelbar angenommen, was dem produktivkreativen Moment in der Begegnung von unterschiedlichen Sinnangeboten Vorschub leistet. Festzuhalten ist, dass ein praxistheoretisches Verständnis von Handeln geeignet ist für die Fragestellung dieses Beitrags, weshalb im Weiteren von sozialen Praktiken gesprochen wird sowie die entwickelten raum- und praxistheoretischen Überlegungen zusammengeführt und für die kulturwissenschaftliche Raumanalyse in den Border Studies fruchtbar gemacht werden.

\section{Räume der Grenze: Analytische Kategorien und Frageperspektiven}

Durch den Fokus auf das körperbasierte Vollzugsgeschehen und den konzeptionellen Brückenschlag zwischen Materialität und Kulturalität setzen sich praxistheoretische Ansätze von traditionellen Handlungserklärungen ab. Damit wird nicht nur die Basis-Überbau-Dichotomie überwunden, ebenso öffnet dies ein Einfallstor für raumbezogene Fragestellungen, die von der Gemachtheit von Räumen ausgehen. Darauf wird von Praxistheoretikern - mit wenigen Ausnahmen ${ }^{48}$ - nur allgemein verwiesen, wie etwa dass „sämtliche soziale Praktiken [sich] als spatializing betrachten lassen und den Raum und dessen Artefakte auf bestimmte Weise organisieren“"49; oder dass soziale Praktiken in ihrer körperlich-materialen Verfasstheit einen activityplace space ${ }^{50}$ konstituieren. Dieser ist dann nicht als ein absolut-substantialistischer Behälter zu denken, sondern als ,ein prozessualer, relationaler Raum der Praktiken und Beziehungen zwischen verkörperten Teilnehmerinnen, Artfakten, Orten und Umgebungen. " ${ }^{\text {51 }}$ Unter methodologischen Gesichtspunkten verweist Schmidt darauf,

47 Vgl. Reckwitz 2003, 291f.; Hörning u. Reuter 2004, 11.

48 z. B. Reckwitz 2012.

49 Ders. 2008, 91.

50 Vgl. Schatzki 2002, 43.

51 Schmidt 2012, 240. 
dass ,im Zuge von Globalisierungsentwicklungen lokale Grenzen [...] kaum noch mit den Grenzen des Sozialen [...] zusammenfallen“ und er schlägt Verfahren vor, „die die verschiedenen Kontexte der Beobachtungsgegenstände abschreiten und den Ver-

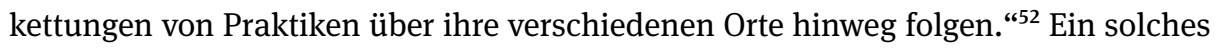
multilokales Vorgehen erschließe dann seine Gegenstände über die verschiedenen Orte und Schauplätze, kartografiere die Terrains und folge den Objekten und Subjekten in ihren Bewegungen.

Damit sind bereits zentrale Aspekte der praxistheoretischen Raumanalyse angesprochen, die konzeptionell aufzufächern und weiter zu vertiefen sind. Dies erfolgt über das Modell Räume der Grenze, das sich nicht als eine Theorie grenzüberschreitenden Handelns verstanden wissen will. Vielmehr geht es darum, eine an raum- und praxistheoretischen Überlegungen orientierte Systematik von heuristischen Kategorien bereitzustellen, die Frageperspektiven eröffnen für die Analyse von Raumkonstitutionen in grenzüberschreitenden Bezügen. Die Erläuterung solcher Kategorien ist nicht zwangsläufig an die hier vorgenommene argumentative Abfolge (a bis d) gebunden.

a. Dem sozial-konstitutiven Raumbegriff folgend werden Räume in sozialen Praktiken hervorgebracht, womit das alltägliche, situationsgebundene ,doing‘ in den Blick gerät. Zugriffe auf Raumemergenzen erfolgen dann über analytische Nahblicke auf die Lebenswirklichkeiten bzw. Praktikenkomplexe von Subjekten. Das bedeutet, dass die multilokal verstreuten sozialen Praktiken von ,Grenzpendlern“ zu untersuchen sind, um die darin hervorgebrachten Räume der Grenze bestimmen zu können. Dafür bieten die besprochenen Raumbegriffe und die praxistheoretische Perspektive geeignete Ansatzpunkte: Wird zunächst der materiale Aspekt von Räumen der Grenze berücksichtigt, so kann die relationale Raumvorstellung aufgegriffen werden, nach der sich Raum aus den Beziehungsstrukturen zwischen Körpern und Artefakten ableitet. Aus praxistheoretischer Sicht wären dann die grenzüberschreitenden Praktiken von ,Grenzpendlern` hinsichtlich der beteiligten bzw. der in den Praktiken in Anordnung gebrachten Körper und Artefakte zu befragen. Ein solches Vorgehen, das auf die bedingenden und ermöglichenden physisch-materialen Aspekte von Räumen der Grenze abstellt, berücksichtigt die Körperlichkeit und Materialität von sozialen Praktiken in ihrer räumlichen Strukturiertheit.

b. Ebenso ist hinsichtlich der mentalen Dimension von Räumen der Grenze eine Konvergenz von raum- und praxistheoretischen Überlegungen auszumachen. Denn während der sozial-konstitutive Raumbegriff die - sich erst im Umgang mit Körpern und Artefakten konstituierende - Bedeutung von Materialitäten betont, ist in den sozialen Praktiken das im Vollzug mobilisierte und sich aktualisierende praktische Wissen zentral. Beide Kategorien fokussieren auf Prozesse der Sinn-

52 Ebd., 256. 
deutung und Sinnzuweisung in der Auseinandersetzung mit der materialen und sozialen Umwelt. Mit Bongaerts kann hier von einem inkorporiert-praktischen Sinn gesprochen werden, der über den körperlichen Vollzug von sozialen Praktiken zum Ausdruck kommt und inter-subjektiv wirksam wird, „ohne dass [...] die Akteure die Sinnhaftigkeit des Verhaltens reflexiv-bewusst geplant hätten oder sie ihnen in objektiv repräsentierter Form zugänglich wäre.“"53 Damit wird der implizite Charakter von praktischem Wissen angesprochen, das sich im Handlungsvollzug konstituiert und in der praxislogischen (Dis-)Kontinuität der körperlich und material verankerten Praktiken manifestiert. Mit Blick auf Räume der Grenze wären dann die sozialen Praktiken von ,Grenzpendlern' hinsichtlich der darin artikulierten Praxislogiken - hier als Formen der Sinndeutungen und Sinnzuschreibung - zu befragen, die im Allgemeinen und in grenzüberschreitenden Bezügen im Besonderen als kontingent anzunehmen sind.

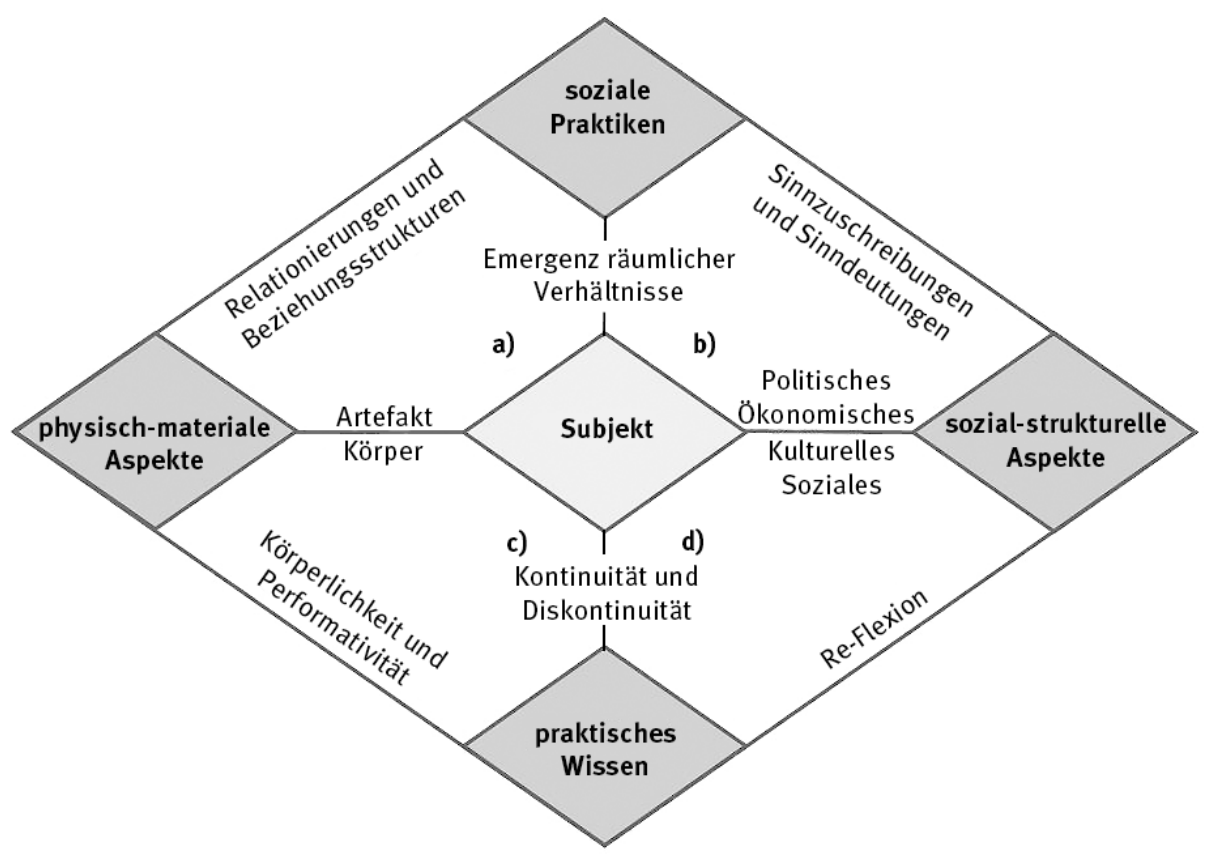

Abb. 1: Räume der Grenze - Heuristik zur praxistheoretischen Beschreibung und Analyse von Raumkonstitutionen in grenzüberschreitenden Bezügen. (Die Gliederung von a bis d gibt keine logische Folge vor, sondern hilft die Abbildung mit dem erläuternden Text zu verknüpfen.)

53 Bongaerts 2012, 23. 
c. Dabei ist Kontingenz nicht zu überhöhen als ein arbiträres Moment, sind Subjekte doch stets als Kreuzungspunkte von historisch überformten und kulturell spezifischen Praktiken zu verstehen. ${ }^{54}$ Daher sind die bedingenden und ermöglichenden Momente sozialer Praktiken gleichermaßen zu berücksichtigen, die nicht - wie soziale Strukturen - außerhalb der Praktiken, sondern ,als stetig erneuerte Voraussetzungen und Resultate von Praktiken“"55 in den Praktiken selbst angesiedelt sind. Im Modell Räume der Grenze werden damit sozial-strukturelle Aspekte angesprochen, die soziale, kulturelle, politische und ökonomische Effekte betreffen und in Praktiken sowohl stabilisiert als auch verändert werden. Dieser Doppelcharakter wird begrifflich als Re-Flexion gefasst: die Kontinuität sozialer Praktiken und damit verbunden die Rückbezüglichkeit bzw. Reproduktion sozial-struktureller Aspekte einerseits (Reflexion) sowie die Diskontinuität sozialer Praktiken und damit verknüpft die ,Beugung‘ bzw. Verschiebung sozialstruktureller Aspekte andererseits (Flexion). Die soziale Praktik ist dann zu verstehen ,[...] als individualistische Strategie oder als gesellschaftliche Routine, als bewusste oder mechanische Aktion, als selbstständige Interpretation oder als Regelerfüllung. " 56 Für die Analyse von Räumen der Grenze ist besonders der flexive Charakter von sozialen Praktiken hervorzuheben, hilft er doch die Destabilisierungen von sozial-strukturellen Aspekten und die produktiv-kreativen Momente der Sinndeutung und Sinnzuweisung theoretisch zu fassen.

d. Der inkorporiert-praktische Sinn - als ein zentrales Moment des praktischen Wissens - ist auf unterschiedliche Weise mit Materialitäten verknüpft, womit erneut die physisch-materialen Aspekte von Räumen der Grenze aufgerufen werden. Es geht dabei um die körperlichen Aufführungen von Praktiken, in der Zeichen prozessiert und Kompetenz demonstriert werden und die für die beteiligten Körper bzw. Subjekte einen praktischen Sinn machen. ${ }^{57}$ Diese als korporalisierende Performativität ${ }^{58} \mathrm{zu}$ fassende Eigenschaft stellt die Ereignishaftigkeit des „körperlichen Dramas“59 sowie das emergente Wechselverhältnis zwischen vollziehendem und beobachtendem Körper in den Mittelpunkt. Letzteres kann Auskunft geben über die Wahrnehmbarkeit von sozialen Praktiken und ihrer sozialen Verstehbarkeit, von der auch unter (gesteigerten) Kontingenzbedingungen - wie etwa im grenzüberschreitenden Kontext - ausgegangen wird. Während über die Körperlichkeit und Performativität die Sinndimension des sozial-konstitutiven Raumbegriffs hier hinsichtlich der inter-subjektiven Struktur eine

54 Vgl. Reckwitz 2009, 176; Reuter 2004, 246.

55 Schmidt 2012, 202.

56 Hörning u. Reuter 2004, 14.

57 Vgl. Schmidt 2012, 59f.; Reckwitz 2010, 190.

58 Vgl. Krämer 2004, 17.

59 Gugutzer 2004, 95. 
Konzeptionalisierung erfährt, gilt es, sich ebenso der inter-objektiven Struktur von sozialen Praktiken zuzuwenden. Dabei geht es um Objekte und Artefakte, die in sozialen Praktiken kompetent eingesetzt werden, und um die materialen Voraussetzungen dafür, dass bestimmte Praktiken erst entstehen und vollzogen werden können. ${ }^{60}$ Die Bedeutungen und praxislogischen Verwendungsweisen von Objekten und Artefakten gehen zum einen von ihnen selbst (als Anrufung...), zum anderen von den hantierenden Körpern (...von praktischem Wissen) aus: „Sie [die Artefakte] werden gehandhabt und drängen sich auf, sie sind Gegenstand der Verwendung und Benutzung und zugleich beeinflussen sie die Form, die soziale Praktiken überhaupt haben können. “61 Die Frage nach dem praktischen Sinn von Objekten und Artefakten führt somit wiederum zu einem emergenten Wechselverhältnis zwischen belebten und nicht-belebten Trägern von sozialen Praktiken und bleibt empirisch zu beantworten.

Festzuhalten ist, dass sich die entwickelte Heuristik in zwei miteinander verwobene Dimensionen gliedert, deren Kreuzungspunkt das als autonom und dezentriert angenommene Subjekt bzw. hier der ,Grenzpendler“ bildet. Die herausgearbeiteten Kategorien sind jeweils auf vielfältige Weise miteinander verwoben und wirken im Vollzugsgeschehen zusammen; ihre analytische Trennung jedoch eröffnet Frageperspektiven und hilfreiche Zugänge zur Beschreibung und Analyse von Räumen der Grenze. So können die an sozialen Praktiken beteiligten Artefakte und Körper - als physischmateriale Aspekte grenzüberschreitender Raumkonstitution - hinsichtlich ihrer Anordnungen befragt werden. Die darüber bestimmbar werdenden Räume können sich grenzüberschreitend aufspannen und spiegeln die relationierenden Praktiken des grenzüberschreitenden Pendelns in ihrer räumlichen Strukturiertheit zurück. Daneben können Artefakte und Körper unter dem performativen Aspekt untersucht werden, womit Fragen nach der Inter-Subjektivität und Inter-Objektivität sowie die damit verbundenen Fragen nach den in grenzüberschreitenden Bezügen hervorgebrachten Bedeutungen und sozialen (Un-)Ordnungen in den Blick kommen. Diese können weiter unter dem Aspekt der Re-Flexion dahingehend befragt werden, inwiefern politische, ökonomische, kulturelle oder soziale Effekte grenzüberschreitende Praktiken beeinflussen bzw. inwiefern letztere die sozial-strukturellen Aspekte verändern.

Die Liste der möglichen Frageperspektiven für analytische Zugriffe auf Räume der Grenze ließe sich weiter fortsetzen und ist je nach Untersuchungsgegenstand spezifisch auszubuchstabieren. Dabei gilt es, besonders die Verknüpfungen zwischen den entwickelten Kategorien in den Blick zu nehmen, leisten sie doch die Verbindungen zwischen Kultur und Materialität und eröffnen räumliche Perspektiven auf soziale

60 Vgl. Fischer-Lichte 2012, 161ff.; Reckwitz 2003, 291.

61 Reckwitz 2010, 193. 
Phänomene in grenzüberschreitenden Bezügen. Aussagen über praxistheoretisch definierte Räume können dann immer nur Aussagen über ihre kulturellen und gleichermaßen materialen Konstitutionsprozesse sein, die sich auf die jeweils betrachteten Wechselwirkungen bzw. Teilaspekte von sozialen Praktiken beziehen. So wurden z. B. grenzüberschreitende Berufspendler und die im Praktikenkomplex ,grenzüberschreitendes Pendeln' hervorgebrachten, räumlichen Verhältnisse untersucht. ${ }^{62}$ Die behandelten Fragestellungen fokussierten auf die sozialen Praktiken in ihren jeweiligen sinnhaften und materialen Dimensionen sowie in ihrer grenzüberschreitenden Multilokalität: die Fahrt zur Arbeit, Zusammenarbeit mit Kollegen, fremdsprachliche Kommunikation, Alltagspraktiken, Vergemeinschaftungspraktiken u. v. m. Die Zusammenschau der Ergebnisse zeigte, dass sich die zu nationalen Grenzen quer liegenden Räume der Grenze durch Merkmale kennzeichnen wie Pluralität, Persistenz, Informalität, Konfliktualität, Kontingenz und Ambivalenz. ${ }^{63}$ Empirisch zu prüfen wäre, inwiefern diese Merkmale auch Räume der Grenze charakterisieren, wie sie z. B. Saisonarbeiter, entsandte Manager oder andere (mobile) borderlands ${ }^{64}$ hervorbringen. Eine Studie $^{65}$ über Wohnmigration greift diese Frage auf. Darin wird die Konstitution von Räumen der Grenze durch Luxemburger untersucht, die ins benachbarte Ausland umziehen und deren Lebenswirklichkeiten - so die These - dies- und jenseits bzw. ,auf' der Grenze anzusiedeln sind. Praxistheoretische Betrachtungen scheinen hier vielversprechend, um kulturelle und gleichermaßen materiale Konstitutionsprozesse von Räumen der Grenze zu erfassen.

\section{Schlussbemerkung}

In Anknüpfung an sozialgeographische und kultursoziologische Überlegungen wurde in diesem Beitrag ein praxistheoretischer Raumbegriff entwickelt und in analytische Kategorien überführt. Anlass für die Ausarbeitung des Modells Räume der Grenze gab die ,Raumblindheit" in den kulturwissenschaftlichen Border Studies, die Raum als theoretische Kategorie weitgehend ausblenden und als geopolitische Ordnungskategorie voraussetzen. Dieser Umstand erscheint paradox, haben die Border Studies doch erst durch die Infragestellungen von räumlichen ,Gegebenheiten“ (z. B. Schengen-Abkommen, Fall des Eisernen Vorhangs) eine bemerkenswerte Entwicklung

62 Vgl. Wille 2012.

63 Vgl. ebd. und ders. 2013.

64 Boeckler 2012.

65 Teilstudie „Regionalisierungen und Identitätskonstruktionen im Kontext grenzüberschreitender Wohnmobilität am Beispiel Luxemburgs“ (Elisabeth Boesen, Gregor Schnuer und Christian Wille) im Rahmen des Projekts „IDENT2 - Regionalisierungen als Identitätskonstruktionen in Grenzräumen“ (2011-2014, Universität Luxemburg). 
durchlaufen. Die Bandbreite der Untersuchungsgegenstände leitet sich zu einem großen Teil aus den oben besprochenen Enträumlichungen ab, ihre raumtheoretische Rückbindung findet aber kaum statt. Das Modell Räume der Grenze leistet hier einen doppelten Beitrag: Es ist ein analytisches Angebot, um Enträumlichung unter dem Aspekt der Verräumlichung zu thematisieren. So ist das Modell auf Phänomene der Enträumlichung anwendbar (z. B. ,Grenzpendler`), die im Doing Culture wiederum neue räumliche Verhältnisse hervorbringen.

Um solche Prozesse sichtbar zu machen, wurde ein Raumbegriff entwickelt, der auf Kultur und Materialität gleichermaßen abstellt und versucht, die theoretisch nicht einholbaren Prozesse des grenzüberschreitenden Geographie-Machens nachzuzeichnen. Er fungiert als Konstrukt für sich fortlaufend verändernde Zusammenhänge zwischen Sinnelementen, Artefakten, Körpern und die räumlichen Ordnungen ihrer Manifestationen. Damit ist offengelegt, dass das Modell Räume der Grenze nicht ausschließlich für die raumsensible Beschreibung und Analyse von sozialen Praktiken in grenzüberschreitenden Bezügen nützlich ist, sondern grundsätzlich dort appliziert werden kann, wo die räumliche Dimension von Praktiken interessiert. Jedoch erweist es sich für die Untersuchung von grenzüberschreitend verstreuten Praktikenkomplexen als besonders gewinnbringend: emanzipiert sich der praxistheoretische Raumbegriff doch von vorgefertigten und übersubjektiv existierenden Wissensstrukturen, ebenso wie von georäumlich definierten Gültigkeitsbereichen normativer Ordnungen. Dies ermöglicht einerseits kontingente Sinnzusammenhänge und ihre Materialisierungen konzeptionell und empirisch $\mathrm{zu}$ fassen, andererseits den Raum in seiner Gemachtheit sowie losgelöst von geopolitischen Grenzziehungen und den damit verbundenen Containerraumvorstellungen zu denken und empirisch zu untersuchen.

\section{Literaturverzeichnis}

Bachmann-Medick (2007): Doris Bachmann-Medick, Cultural Turns. Neuorientierungen in den Kulturwissenschaften, Reinbek, (2. Aufl.), 284-327.

Bathelt u. Glückler (2003): Harald Bathelt u. Johannes Glückler, Wirtschaftsgeographie.

Ökonomische Beziehungen in räumlicher Perspektive, Stuttgart.

Boeckler (2012): Marc Boeckler, „Borderlands“, in: Nadine Marquardt u. Verena Schreiber (Hgg.), Ortsregister. Ein Glossar zu Räumen der Gegenwart, Bielefeld, 44-49.

Bonacker (2008): Thorsten Bonacker, „Gesellschaft. Warum die Einheit der Gesellschaft aufgeschoben wird“, in: Stephan Moebius u. Andreas Reckwitz (Hgg.), Poststrukturalistische Sozialwissenschaften, Frankfurt/M., 27-42.

Bongaerts (2012): Gregor Bongaerts, Sinn, Bielefeld.

Döring u. Thielmann (2008): Jörg Döring u. Tristan Thielmann (Hgg.), Spatial Turn. Das Raumparadigma in den Kultur- und Sozialwissenschaften, Bielefeld.

Einstein (1960): Albert Einstein, „Vorwort“, in: Max Jammer (Hg.), Das Problem des Raumes. Die Entwicklungen der Raumtheorien, (Bd. 8), Darmstadt, 7-17.

Fischer-Lichte (2012): Erika Fischer-Lichte, Performativität. Eine Einführung, Bielefeld. 
Gugutzer (2004): Robert Gugutzer, Soziologie des Körpers, Bielefeld.

Günzel (2010): Stephan Günzel (Hg.), Raum. Ein interdisziplinäres Handbuch, Stuttgart.

Hörning u. Reuter (2004): Karl H. Hörning u. Julia Reuter, „Doing Culture. Kultur als Praxis“, in: Karl H. Hörning u. Julia Reuter (Hgg.), Doing Culture. Neue Positionen zum Verhältnis von Kultur und sozialer Praxis, Bielefeld.

Kajetzke u. Schroer (2010): Laura Kajetzke u. Markus Schroer, „Sozialer Raum. Verräumlichung“, in: Stephan Günzel (Hg.), Raum. Ein interdisziplinäres Handbuch, Stuttgart, 192-203.

Kajetzke u. Schroer (2012): Laura Kajetzke u. Markus Schroer, „Space Studies“, in: Stephan Moebius

(Hg.), Kultur. Von den Cultural Studies bis zu den Visual Studies. Eine Einführung, Bielefeld, 196-215.

Krämer (2004): Sybille Krämer, „Was haben ,Performativität‘ und ,Medialität‘ miteinander zu tun? Plädoyer für eine in der ,Aisthetisierung gründende Konzeption des Performativen“, in: Sybille Krämer (Hg.), Performativität und Medialität, München, 11-32.

Lefebvre (1991): Henri Lefebvre, The production of space, Oxford.

Lippuner (2005): Roland Lippuner, Raum - Systeme - Praktiken. Zum Verhältnis von Alltag, Wissenschaft und Geographie, (Sozialgeographische Bibliothek, Bd. 2), Stuttgart.

Löw (2001): Martina Löw, Raumsoziologie, Frankfurt/M.

Mau (2007): Steffen Mau, Transnationale Vergesellschaftung. Die Entgrenzung sozialer Lebenswelten, Frankfurt/M.

Moebius (2008): Stephan Moebius, „Handlung und Praxis. Konturen einer poststrukturalistischen Praxistheorie“, in: Stephan Moebius u. Andreas Reckwitz (Hgg.), Poststrukturalistische Sozialwissenschaften, Frankfurt/M., 58-74.

Moebius (2012a): Stephan Moebius (Hg.), Kultur. Von den Cultural Studies bis zu den Visual Studies. Eine Einführung, Bielefeld.

Moebius (2012b): Stephan Moebius, „Kulturforschungen der Gegenwart - die Studies. Einleitung“, in: Stephan Moebius (Hg.), Kultur. Von den Cultural Studies bis zu den Visual Studies. Eine Einführung, Bielefeld, 7-12.

Newton (1988): Isaac Newton, Mathematische Grundlagen der Naturphilosophie, (hg. von Ed Dellian), Hamburg, orig. 1687.

Pries (2008): Ludger Pries, Die Transnationalisierung der sozialen Welt, Frankfurt/M.

Ratzel (1966): Friedrich Ratzel, Der Lebensraum. Eine biogeographische Studie, Darmstadt.

Reckwitz (2003): Andreas Reckwitz, „Grundelemente einer Theorie sozialer Praktiken. Eine sozialtheoretische Perspektive“, Zeitschrift für Soziologie 32 (4), 282-301.

Reckwitz (2004): Andreas Reckwitz, „Die Entwicklung des Vokabulars der Handlungstheorien. Von den zweck- und normorientierten Modellen zu den Kultur- und Praxistheorien“, in: Manfred Gabriel (Hg.), Paradigmen der akteurszentrierten Soziologie, Wiesbaden, 303-328.

Reckwitz (2008): Andreas Reckwitz, „Subjekt/Identität“, in: Stephan Moebius u. Andreas Reckwitz (Hgg.), Poststrukturalistische Sozialwissenschaften, Frankfurt/M., 75-92.

Reckwitz (2009): Andreas Reckwitz, „Praktiken der Reflexivität. Eine kulturtheoretische Perspektive auf hochmodernes Handeln“, in: Fritz Böhle u. Margit Weihrich (Hg.), Handeln unter Unsicherheit, Wiesbaden, 169-182.

Reckwitz (2010): Andreas Reckwitz, „Auf dem Weg zu einer kultursoziologischen Analytik zwischen Praxeologie und Poststrukturalismus“, in: Monika Wohlrab-Sahr (Hg.), Kultursoziologie. Paradigmen - Methoden - Fragestellungen, Wiesbaden, 179-205.

Reckwitz (2012): Andreas Reckwitz, „Affective spaces: a praxeological outlook“, Rethinking history 16 (2), 241-258.

Reuter (2004): Julia Reuter, „Postkoloniales Doing Culture. Oder: Kultur als translokale Praxis“, in: Karl H. Hörning u. Julia Reuter (Hgg.), Doing Culture. Neue Positionen zum Verhältnis von Kultur und sozialer Praxis, Bielefeld, 239-255. 
Schatzki (2002): Theodore Schatzki, The Site of the Social. A Philosophical Account of the Constitution of Social Life and Change, Pennsylvania State University.

Schatzki (2010): Theodore Schatzki, The Timespace of Human Activity. On Performance, Society, and History as Indeterminate Teleological Events, Lexington.

Schmidt (2012): Robert Schmidt, Soziologie der Praktiken. Konzeptionelle Studien und empirische Analysen, Frankfurt/M.

Schmitt-Egner (2005): Peter Schmitt-Egner, Handbuch zur Europäischen Regionalismusforschung. Theoretisch-methodische Grundlagen, empirische Erscheinungsformen und strategische Optionen des Transnationalen Regionalismus im 21. Jahrhunderts, Wiesbaden.

Schroer (2006): Markus Schroer, Räume, Orte, Grenzen. Auf dem Weg zu einer Soziologie des Raums, Frankfurt/M.

Schroer (2008): Markus Schroer, „,Bringing space back in“ - Zur Relevanz des Raums als soziologische Kategorie“, in: Jörg Döring u. Tristan Thielmann (Hgg.), Spatial Turn. Das Raumparadigma in den Kultur- und Sozialwissenschaften, Bielefeld, 125-148.

Schütz (1972): Alfred Schütz, „Der Fremde. Ein sozialpsychologischer Versuch“, in: Alfred Schütz (Hg.), Gesammelte Aufsätze (Studien zur soziologischen Theorie, Bd. 2), Den Haag, (1944), 43-69.

Van Houtum, Kramsch u. Zierhofer (2005): Henk van Houtum, Olivier Kramsch u. Wolfgang Zierhofer (Hgg.), B/Ordering Space, Aldershot.

Van Houtum u. van Naerssen (2002): Henk van Houtum u. Ton van Naerssen, „Bordering, Ordering and Othering“, Journal of Economic and Social Geography 93 (2), 125-136.

Walter-Wastl (2011): Doris Walter-Wastl (Hg.), The Ashgate Research Companion to Border Studies, Farnham.

Weichhart (2008): Peter Weichhart, Entwicklungslinien der Sozialgeographie. Von Hans Bobek bis Benno Werlen (Sozialgeographie kompakt, Bd. 1), Stuttgart.

Werlen (1997): Benno Werlen, Sozialgeographie alltäglicher Regionalisierungen. Globalisierung, Region und Regionalisierung (Bd. 2), (Erdkundliches Wissen, Bd. 119), Stuttgart.

Werlen (1999): Benno Werlen, Sozialgeographie alltäglicher Regionalisierungen. Zur Ontologie von Gesellschaft und Raum, (Bd. 1), Stuttgart.

Werlen (2004): Benno Werlen, Sozialgeographie. Eine Einführung, Bern, (2. Aufl.).

Werlen (2007a): Benno Werlen, Globalisierung, Region und Regionalisierung. Sozialgeographie alltäglicher Regionalisierungen, (Bd. 2), Stuttgart, (2. Aufl.).

Werlen (2007b): Benno Werlen, „Einleitung“, in: Benno Werlen (Hg.), Sozialgeographie alltäglicher Regionalisierungen. Ausgangspunkte und Befunde empirischer Forschung, (Bd. 3), (Erdkundliches Wissen, Bd. 121), Stuttgart, 9-16.

Werlen (2008a): Benno Werlen, „Körper, Raum und mediale Repräsentation“, in: Jörg Döring u. Tristan Thielmann (Hgg.), Spatial Turn. Das Raumparadigma in den Kultur- und Sozialwissenschaften, Bielefeld, 365-392.

Werlen (2008b): Benno Werlen, Sozialgeographie. Eine Einführung, Bern, (3. Aufl.).

Werlen (2009): Benno Werlen, „Geographie/Sozialgeographie“, in: Stephan Günzel (Hg.), Raumwissenschaften, Frankfurt/M., 142-158.

Werlen (2010): Benno Werlen, Gesellschaftliche Räumlichkeit. Orte der Geographie, (Bd. 1), Stuttgart.

Wille (2012): Christian Wille, Grenzgänger und Räume der Grenze. Raumkonstruktionen in der Großregion SaarLorLux (Luxemburg-Studien / Etudes luxembourgeoises, Bd. 1), Frankfurt/M.

Wille (2013): Christian Wille, „Zur Persistenz und Informalität von Räumen der Grenze. Theoretischkonzeptionelle Überlegungen und empirische Befunde“, Itinera - Schweizerische Zeitschrift für Geschichte (Beiheft „Hier und dort. Ressourcen und Verwundbarkeiten in multilokalen Lebenswelten / Ici et là. Ressources et vulnérabilités dans la vie multilocale“, hrsg. von Cédric Duchêne-Lacroix und Pascal Maeder), Nr. 34, 2013, 99-112. 